\title{
BMJ Open Anticoagulant therapies for acute venous thromboembolism: a comparison between those discharged directly from the emergency department versus hospital in two Canadian cities
}

\author{
Tammy J Bungard, ${ }^{1}$ Bruce Ritchie, ${ }^{2}$ Jennifer Bolt, ${ }^{3}$ William M Semchuk ${ }^{3}$
}

To cite: Bungard TJ, Ritchie B, Bolt J, et al. Anticoagulant therapies for acute venous thromboembolism: a comparison between those discharged directly from the emergency department versus hospital in two Canadian cities. BMJ Open 2018;8:e022063. doi:10.1136/ bmjopen-2018-022063

- Prepublication history for this paper is available online. To view these files, please visit the journal online (http://dx.doi org/10.1136/bmjopen-2018022063).

Received 30 January 2018 Revised 14 August 2018 Accepted 28 August 2018

\section{SLinked}

- http://dx.doi.org/10.1136/ bmjopen-2018-022064

- http://dx.doi.org/10.1136/ bmjopen-2018-022065

Check for updates

(C) Author(s) (or their employer(s)) 2018. Re-use permitted under CC BY-NC. No commercial re-use. See rights and permissions. Published by BMJ.

${ }^{1}$ Division of Cardiology, University of Alberta, Edmonton, Alberta, Canada

${ }^{2}$ Division of Hematology, University of Alberta, Edmonton, Alberta, Canada

${ }^{3}$ Pharmacy Services, Regina Qu'Appelle Health Region, Regina, Saskatchewan, Canada

Correspondence to

Dr Tammy J Bungard;

tammy.bungard@ualberta.ca

\section{ABSTRACT}

Objective To compare the characteristics/management of acute venous thromboembolism (VTE) for patients either discharged directly from the emergency department (ED) or hospitalised throughout a year within two urban cities in Canada.

Design Retrospective medical record review.

Setting Hospitals in Edmonton, Alberta $(n=4)$ and Regina, Saskatchewan $(n=2)$ from April 2014 to March 2015.

Participants All patients discharged from the ED or hospital with acute deep vein thrombosis or pulmonary embolism (PE). Those having another indication for anticoagulant therapy, pregnant/breast feeding or anticipated lifespan $<3$ months were excluded.

Primary and secondary outcomes Primarily, to compare proportion of patients receiving traditional therapy (parenteral anticoagulant \pm warfarin) relative to a direct oral anticoagulant (DOAC) between the two cohorts. Secondarily, to assess differences with therapy selected based on clot burden and follow-up plans postdischarge.

Results 387 (25.2\%) and 665 (72.5\%) patients from the ED and hospital cohorts, respectively, were included. Compared with the ED cohort, those hospitalised were older (57.3 and 64.5 years; $p<0.0001$ ), more likely to have PE (35.7\% vs $83.8 \%)$ with a simplified Pulmonary Embolism Severity Index (sPESI) $\geq 1$ (31.2\% vs $65.2 \%$ ), cancer $(14.7 \%$ and $22.3 \% ; p=0.003)$ and pulmonary disease $(10.1 \%$ and $20.6 \% ; p<0.0001)$. For the ED and hospital cohorts, similar proportions of patients were prescribed traditional therapies $(72.6 \%$ and $71.1 \%)$ and a DOAC (25.8\% and $27.4 \%$, respectively). For the ED cohort, DOAC use was similar between those with a sPESI score of 0 and $\geq 1$ ( $35.1 \%$ and $34.9 \%, p=0.98$ ) whereas for those hospitalised lower risk patients were more likely to receive a DOAC $(31.4 \%$ and $23.8 \%, p<0.055)$. Follow-up was most common with family physicians for those hospitalised $(51.5 \%)$, while specialists/VTE clinic was most common for those directly discharged from the ED (50.6\%).

Conclusions Traditional and DOAC therapies were proportionately similar between the ED and hospitalised cohorts, despite clear differences in patient populations and follow-up patterns in the community.
Strengths and limitations of this study

- Over the span of a complete year, chart reviews were performed on all eligible patients, enabling accurate data capture based on documentation in the institution-based chart.

- The study design enabled comparison of care across the same time interval, between those discharged directly from the emergency department versus those being hospitalised.

- Including two urban centres from different Canadian provinces offers more generalisable data within the Canadian environment.

- The study design limited us to collect data from institution-based charts and prevented us from following patients after leaving the institutions, thereby prohibiting the assessment of patient outcomes.

\section{INTRODUCTION}

Venous thromboembolism (VTE), encompassing deep vein thrombosis (DVT) and pulmonary embolism (PE), is a common disease process affecting up to $5 \%$ of the population. ${ }^{1}$ Given that $\mathrm{PE}$ is associated with a higher short-term mortality than DVT, management may differ in terms of direct discharge from the emergency department (ED) versus admission to hospital as well as in the selection of anticoagulant therapy. While guidelines put forth in 2012 recommended the treatment of DVT at home over treatment in hospital, only more recent guidelines (2016) have suggested that patients having a low-risk PE (defined as a Pulmonary Embolism Severity Index (PESI) score $<85$ or simplified score of 0 ) should be managed at home or have an early discharge over standard discharge (after the first 5 days of treatment). ${ }^{23}$ Clinical practices comparing patient characteristics for those directly discharged from the ED versus admitted to hospital 
Table 1 Timing of approval of direct oral anticoagulants in Canada

\begin{tabular}{ll}
\hline Agent-indication & Health Canada approval \\
\hline $\begin{array}{l}\text { Rivaroxaban-deep vein } \\
\text { thrombosis }\end{array}$ & February 2012 \\
$\begin{array}{l}\text { Rivaroxaban- pulmonary } \\
\text { embolism }\end{array}$ & April 2013 \\
$\begin{array}{l}\text { Dabigatran - venous } \\
\text { thromboembolism (VTE) }\end{array}$ & June 2014 \\
Apixaban-VTE & November 2014 \\
Edoxaban - VTE & November 2016 \\
\hline
\end{tabular}

within the same institution(s) for an acute VTE presentation have not been reported in the literature.

Within the past 5 years, several direct oral anticoagulants (DOACs), including rivaroxaban, apixaban, dabigatran and edoxaban, have been approved for use for VTE (table 1). ${ }^{4-7}$ All DOACs have been shown to be non-inferior to standard therapy in the acute treatment of VTE with similar or less major bleeding. ${ }^{8-13}$ These DOACs all offer advantages over standard therapy with a parenteral anticoagulant transitioned to warfarin; all DOACs have standardised dosing regimens for this indication that do not require routine coagulation monitoring and oral only therapy with rivaroxaban and apixaban may be prescribed alone for acute VTE. While European guidelines ${ }^{14}$ have yet to differentiate the use of warfarin versus the DOACs for the acute treatment of VTE, North American guidelines now recommend the DOACs to be used among non-cancer patients in preference to warfarin. ${ }^{3}$ While the DOACs offer more straightforward care delivery, use of these agents relative to standard care may vary between patients being discharged directly from the ED versus those being hospitalised. We hypothesised that DOACs would be used more among those discharged directly from the ED versus those hospitalised, given the ease of administration of DOACs and our anticipation that lower risk VTE patients would be discharged directly from the ED. As such, the purpose of this study was to compare the characteristics and management of acute VTE for patients either discharged directly from the ED or hospitalised throughout a year within two urban cities in Canada.

\section{METHODS}

\section{Study design, setting and patients}

A retrospective chart review was conducted at two urban centres including the Edmonton, Alberta, Canada area (two tertiary care teaching hospitals (University of Alberta Hospital, Royal Alexandra Hospital) and two community-based hospitals (Grey Nuns Hospital, Misericordia Hospital)) and the Regina, Saskatchewan, Canada area (two tertiary care hospitals (Regina General Hospital and Pasqua Hospital)). The Edmonton area has a population of 1328290 and is a referral source for central/ Northern Alberta, while Regina has 230020 with a large catchment area of 500000 and serves as the referral point for patients in Southern Saskatchewan. ${ }^{1516}$

Across a 1-year interval (1 April 2014 to 31 March 2015), all patients discharged directly from the ED or following hospitalisation with a DVT (International Classification of Diseases (ICD)-10 code I82 +subindices) or PE (ICD-10 code I26 +subindices) diagnosis were screened. Patients were excluded if they had atypical clot locations given they were not enrolled in recent non-inferiority trials of DOACs, if they required therapeutic anticoagulation for another indication, were $<18$ years of age, were pregnant or breast feeding, died in hospital or had an anticipated lifespan $<3$ months (evidenced by palliation or prognosis documentation).

\section{Objectives}

Our primary objective was to compare the proportion of patients with acute VTE who were prescribed standard therapies (parenteral anticoagulant \pm warfarin) relative to a DOAC between those discharged directly from the ED or being hospitalised. Secondarily, comparisons between the two cohorts were made based on burden of the clot. For DVT, this was defined as proximal or distal, and for PE the simplified PESI (sPESI) was assessed at the point of presentation. ${ }^{17}{ }^{18}$ Lastly, we describe differences in planned follow-up postdischarge from either the ED or hospital. ${ }^{19} 20$

While our objectives herein compare the ED versus hospitalised cohorts in aggregate for the urban settings, separate preplanned analysis specific to the ED cohort and the hospitalised cohort that also encompass rural Alberta have been performed and these companion papers will be published together even all were not accepted on the same date. For each of these manuscripts, the management of acute VTE is detailed, encompassing variation based on geographical setting, DVT versus PE, clot burden, time within institution-based care, follow-up patterns in the respective communities and (where applicable) concordance of DOAC use as per the Canadian product monographs.

\section{Patient and public involvement statement}

Patients and or the public were not involved in this study.

\section{Data sources/grouping and statistical analysis}

Data were extracted based on documentation from the institution-based medical record and entered directly into Research Electronic Data Capture (REDCap). All data within REDCap are at the University of Alberta, and are available to the principal investigator (TJB). No data sharing agreement is in place, hence data sharing is not applicable. Data from Edmonton and Regina were combined to compare management between those discharged directly from the ED and from the hospital. For PE, we combined those having PE alone with those having both PE and DVT.

Data analysis was performed at the Epidemiology Coordinating and Research Centre. The $\chi^{2}$ test was 
Emergency Department Cohort

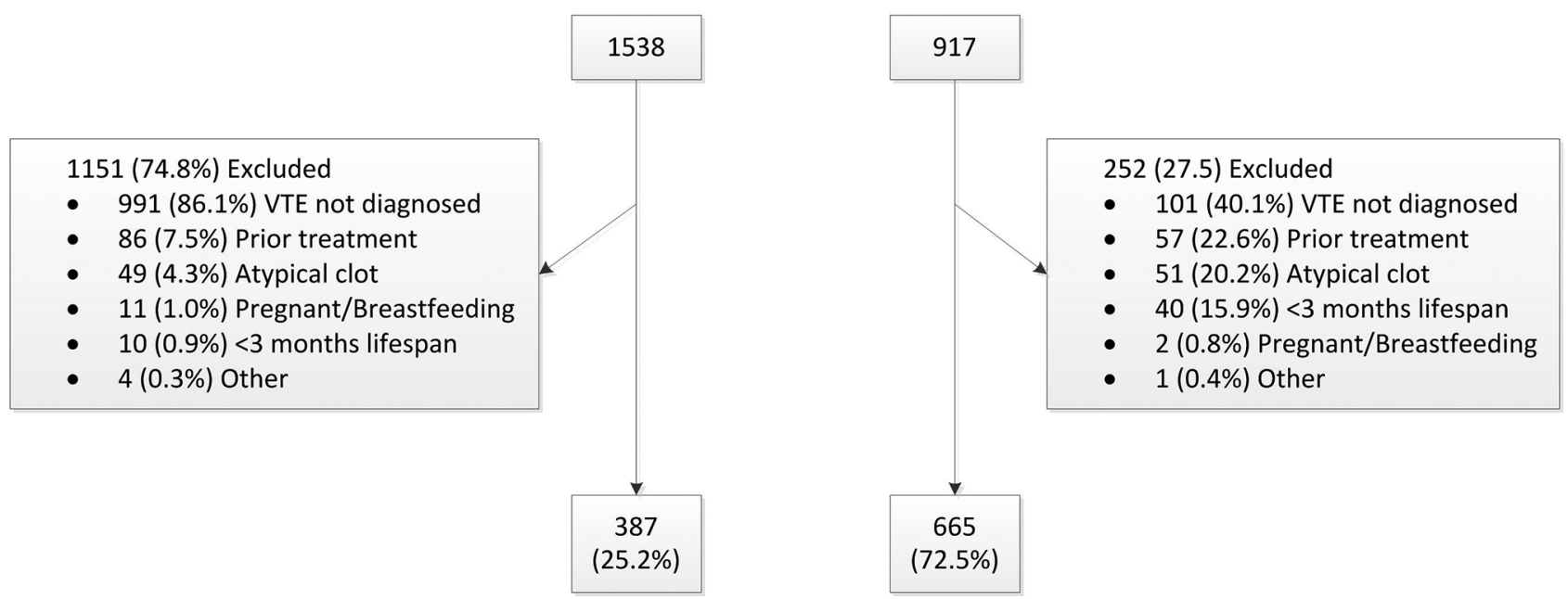

Figure 1 Patient flow in emergency department versus hospitalised cohorts. VTE, venous thromboembolism.

used to compare proportions between the ED and hospital cohorts. Fisher's exact test was used when small expected frequencies were present and hence questioned the validity of the $\chi^{2}$ test. A t-test was used to compare patients' age between cohorts. Non-parametric WilcoxonMann-Whitney test was used to compare patients' weight between cohorts. Logistic regression was used to determine if factors were associated with the use of DOAC versus traditional therapy. Statistical analysis was carried using SAS V.9.4.

\section{RESULTS}

After screening 1538 and 917 charts from the ED and hospital cohorts, $387(25.2 \%)$ and $665(72.5 \%)$ were included, respectively (figure 1 ). The vast majority $(86.1 \%)$ were excluded from the ED given an acute VTE was not diagnosed, whereas in the hospital cohort the most common reasons for exclusions were lack of an acute VTE $(40.1 \%)$, requiring therapeutic anticoagulation for another indication $(22.6 \%)$ and the presence of an atypical clot (20.2\%). Compared with the ED cohort, those admitted to the hospital were older (57.3 and 64.5 years; $\mathrm{p}<0.0001)$ and more likely to have cancer $(14.7 \%$ and 22.3\%; $\mathrm{p}=0.003)$ and pulmonary disease $(10.1 \%$ and $20.6 \% ; \mathrm{p}<0.0001$ ) (table 2). For the ED cohort, the majority were discharged with DVT $(64.3 \%)$ whereas for the hospital cohort the majority had PE $(83.8 \%)$ $(\mathrm{p}<0.0001)$.

Between the ED and hospitalised cohorts, similar proportions of patients were prescribed traditional therapies $(72.6 \%$ and $71.1 \%)$ and a DOAC $(25.8 \%$ and $27.4 \%$, respectively) (table 3). Differences emerged between the type of traditional therapy prescribed, with those leaving the ED being more likely to have a parenteral anticoagulant alone $(42.9 \%$ vs $26.9 \%)$ while those hospitalised more commonly had a parenteral anticoagulant to warfarin-based regimen $(29.7 \%$ for ED and $44.2 \%$ for hospital cohort) $(\mathrm{p}<0.0001)$.

Among those going home with PE, the majority in the ED cohort were low risk or had a sPESI of $0(68.8 \%)$, whereas for the hospital cohort the majority had a sPESI score of $\geq 1 \quad(65.1 \%) \quad(p<0.0001)$. Use of a DOAC for PE for the ED versus hospitalised cohort was not significantly different $(34.8 \%$ and $26.9 \%$, respectively; $\mathrm{p}=0.067)$. For the ED cohort, use of a DOAC was not different among those with a sPESI score of $0(35.1 \%)$ or $\geq 1(34.9 \%)$ $(\mathrm{p}=0.98)$; whereas those discharged from hospital with a sPESI score of 0 were more likely to receive a DOAC compared with those with a score of $\geq 1(31.4 \%$ vs $23.8 \%$, respectively) $(\mathrm{p}<0.055)$. Few patients had distal DVTs in the hospital cohort, limiting comparisons between the two groups. Logistic regression identified less use of DOACs among those with a history of cancer (OR 0.16, $95 \%$ CI 0.082 to $0.32 ; \mathrm{p}<0.0001)$ and recent surgery (OR $0.22,95 \%$ CI 0.074 to $0.63 ; p=0.005$ ). No other factor (age, gender, creatinine clearance $(\mathrm{CrCl})$, PESI score, discharged from the ED or hospitalised) was significantly associated with DOAC versus traditional therapy.

Once discharged, follow-up was to occur more often with the family doctor for the hospital cohort $(51.5 \%)$ compared with the ED cohort (29.7\%). Those discharged directly from the ED were most commonly referred to a specialist/VTE clinic $(50.6 \%)$.

\section{DISCUSSION}

Our study identified similar proportionate use of DOACs with varying traditional therapies (parenteral anticoagulant \pm warfarin) in those being discharged directly from the ED and those hospitalised, despite showing distinct differences in the characteristics of the two cohorts. As expected, relative to the ED cohort, those hospitalised were older, had more comorbidities (cancer, pulmonary 
Table 2 Baseline characteristics ED versus hospital discharge

\begin{tabular}{|c|c|c|c|}
\hline & ED cohort & Hospital cohort & $P$ values \\
\hline Screened (N) & 1538 & 917 & \\
\hline Included N (\%) & $387(25.2)$ & $665(72.5)$ & \\
\hline Male $(\mathrm{N}, \%)$ & $206(53.2)$ & $334(50.2)$ & 0.35 \\
\hline Mean age $($ mean $\pm S D)$ & $57.2 \pm 18.1$ & $63.2 \pm 17.3$ & $<0.0001$ \\
\hline Weight done* & $281(72.6 \%)$ & $652(98.1 \%)$ & \\
\hline Median weight $(\mathrm{kg})$ & $84.0(68.1,100.0)$ & $86.0(70.0,106.0)$ & 0.036 \\
\hline $\mathrm{CrCl}$ done* & $306(79.1 \%)$ & $632(95.0 \%)$ & 0.0008 \\
\hline$<30 \mathrm{~mL} / \mathrm{min}$ & $8(2.6 \%)$ & $22(3.5 \%)$ & \\
\hline $30-49 \mathrm{~mL} / \mathrm{min}$ & $29(9.5 \%)$ & $118(18.7 \%)$ & \\
\hline$>50 \mathrm{~mL} / \mathrm{min}$ & 269 (87.9\%) & $492(77.9 \%)$ & \\
\hline Length of stay (median, IQ range) & 6.17 hours $(4.4,9.0)$ & 6.0 days $(3.0,11.0)$ & - \\
\hline VTE & & & $<0.0001$ \\
\hline DVT & $249(64.3 \%)$ & $108(16.2 \%)$ & \\
\hline Distal DVT & $45(18.1 \%)$ & $13(12.0 \%)$ & \\
\hline $\begin{array}{l}\text { Proximal DVT (footnote double "s" to } \\
\text { go here) }\end{array}$ & $190(76.3 \%)$ & $90(83.3 \%)$ & \\
\hline Not documented & $14(5.6 \%)$ & $5(4.6 \%)$ & \\
\hline PE† and PE+DVT† & $138(35.7 \%)$ & $557(83.8 \%)$ & \\
\hline \multicolumn{4}{|l|}{ History of: } \\
\hline Cancer & $57(14.7 \%)$ & $148(22.3 \%)$ & 0.003 \\
\hline Pulmonary disease & $39(10.1 \%)$ & $137(20.6 \%)$ & $<0.0001$ \\
\hline Prior VTE & $86(22.2 \%)$ & $94(14.1 \%)$ & 0.0008 \\
\hline Recent surgery & $29(7.5 \%)$ & $32(4.8 \%)$ & 0.073 \\
\hline PE-simplified PESI score & & & $<0.0001$ \\
\hline 0 point & $95(68.8 \%)$ & 191 (34.9\%)‡ & \\
\hline$>1$ point & $43(31.2 \%)$ & $357(65.2 \%) \ddagger$ & \\
\hline
\end{tabular}

*Not all patients had weight and serum creatinine documented in the chart.

†PE and PE+DVT are reported together.

$\ddagger$ PESI score could not be calculated in nine patients due to missing variable(s).

$\S$ Combined popliteal, femoral, common femoral and iliac.

$\mathrm{CrCl}$, creatinine clearance; DVT, deep vein thrombosis; ED, emergency department; PE, pulmonary embolism; PESI, Pulmonary Embolism

Severity Index; VTE, venous thromboembolism.

disease, $\mathrm{CrCl}<50 \mathrm{~mL} / \mathrm{min}$ ), were more likely to have $\mathrm{PE}$ and the majority in the hospitalised cohort with $\mathrm{PE}$ had sPESI scores $\geq 1$. Follow-up in the community was different between the two cohorts and influenced by therapy at discharge; the majority hospitalised had oral anticoagulant therapy implemented and were to see their family physicians $(51.5 \%)$ whereas $43 \%$ discharged directly from the ED were prescribed a parenteral anticoagulant alone with follow-up to occur with either a VTE clinic or specialist $(50.6 \%)$.

Recently updated guidelines for VTE management now state patients with low-risk PE and adequate home circumstances may be treated entirely at home, ${ }^{3}$ given the risk of adverse events is low. ${ }^{21-23}$ In our study, the majority with VTE hospitalised had PE (83.8\%), while $35.7 \%$ discharged directly from EDs had PE. Concordant with risk assessment, the majority of patients with
PE going home from the ED $(68.8 \%)$ had a PESI score of 0 , while the majority admitted $(65.2 \%)$ had scores $>1$. A large database review ( $\mathrm{n}=394000 \mathrm{ED}$ visits for $\mathrm{PE}$ ) spanning 2006-2010 reported hospitalisation rates for PE to be $90 \%$ and to not change over time. ${ }^{24}$ Of those admitted, $54 \%$ had sPESI scores of 0 , and the authors suggested that these low-risk patients could be treated out of hospital. A smaller cohort of 175 patients with PE reported $32.0 \%$ to be sent home within 24 hours; $62.5 \%$ of these patients had a sPESI score of $0 .^{25}$ Among those sent home within 24 hours, the majority $(57.1 \%)$ received expedited follow-up in the community (within 3 days). The 30-day adverse outcomes (unscheduled ED visit within 3 days, thromboembolism-related readmission within 5 days or mortality out to 30 days) among those having short versus longer stays ( $>24$ hours) was low and not different between the two groups. These data are similar to ours 
Table 3 Discharge therapies and follow-up

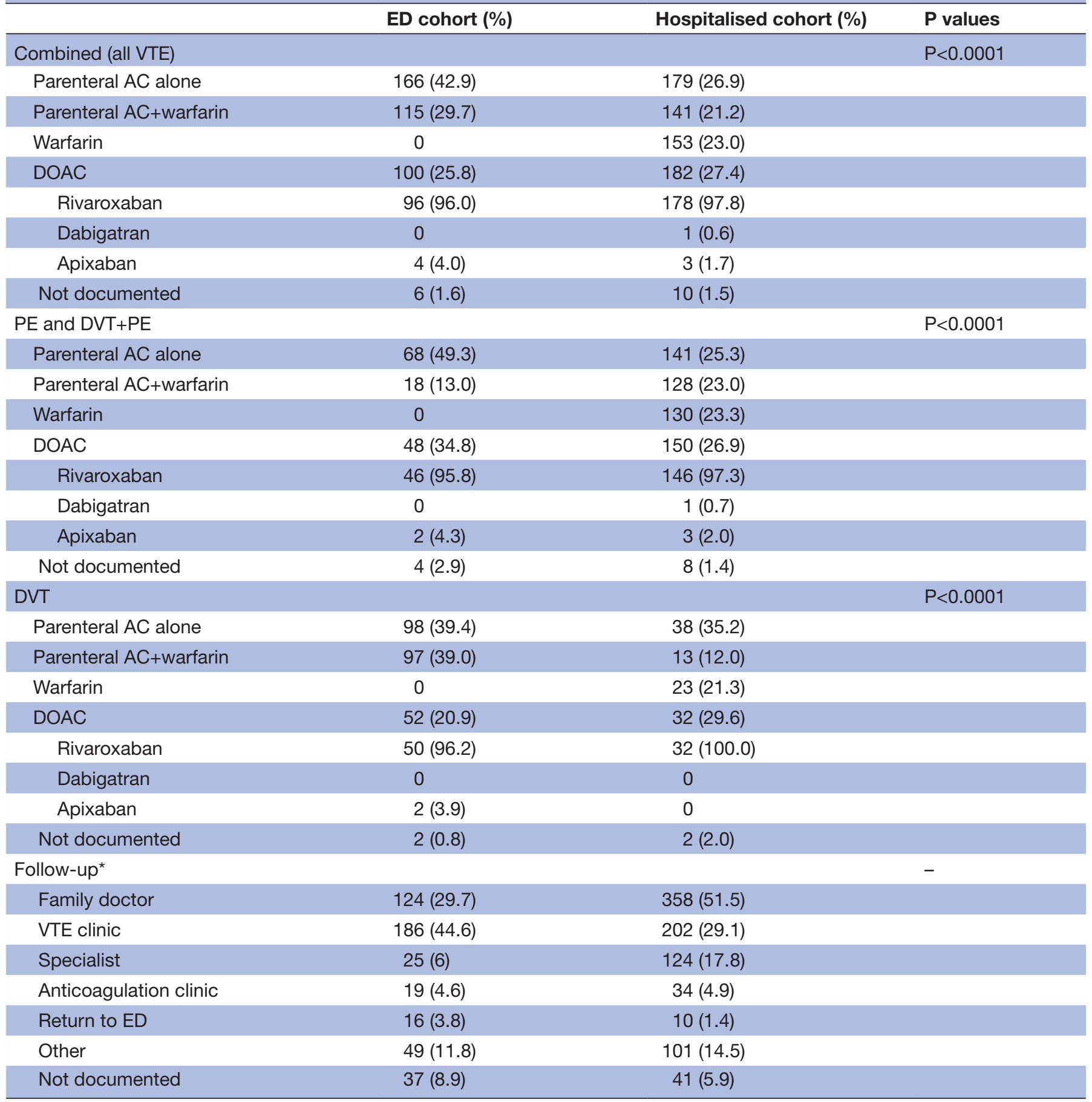

*Not mutually exclusive.

AC, anticoagulant; DOAC, direct oral anticoagulant; DVT, deep vein thrombosis; ED, emergency department; PE, pulmonary embolism; VTE, venous thromboembolism.

in that a similar portion of PE patients were discharged directly from the ED (35.7\%), the majority with PE in our cohort had a PESI score of $0(68.8 \%)$ and follow-up was to occur with either a VTE clinic or specialist (50.6\%).

Our study identified use of a DOAC in $26.8 \%$ of all patients, with similar proportions in the ED and hospitalised cohort. The DOAC used was almost exclusively rivaroxaban $(97.2 \%)$, aligning with this agent being the first approved for the treatment of DVT and PE in
Canada (February 2012 and April 2013, respectively). Other agents were approved either during or after the audit interval (table 1). Use of a parenteral anticoagulant alone at discharge was more common in the ED cohort versus those hospitalised, and attributable to ED patients being referred to VTE clinics/specialists for follow-up and decisions regarding longer term anticoagulant options. As such, our data may underestimate the prevalence of DOAC use for acute VTE. An audit of 
an academic centre in Quebec, Canada also reported a high rate of parenteral anticoagulant use $(61.7 \%)$ as the initial treatment for patients newly diagnosed VTE, with subsequent treatment strategies including warfarin $(54.7 \%)$, parenteral therapy alone $(27.7 \%$; the majority $(85.9 \%)$ had cancer) and rivaroxaban $(17.6 \%) .{ }^{26}$ Lower rates of rivaroxaban were reported in this audit compared with ours, likely attributable to the timing of the audit (February to September 2013) occurring at an interval early in the approval of rivaroxaban. In contrast to the ED cohort in our study, warfarin $( \pm$ parenteral anticoagulant) based regimens were more common in those hospitalised, reflecting decisions for longer term therapy being made with most referred to the family doctor for ongoing follow-up and monitoring.

\section{Strengths and limitations}

Our study does have a few limitations. The retrospective design limited access to only documentation available in the institution-based charts. Hence, certain data elements were missing (eg, weight, creatinine) and are acknowledged and reported herein. Reviewing each chart, however, ensured accurate data capture of our patient population and related data elements. Being limited to data only within the institution-based chart prohibited us from following these patients after leaving the institution, thereby preventing us from tracking longer term outcome data. Second, given the scope of this study included two cities with multiple sites, three data extractors were needed to complete the audits. A single extractor in Edmonton trained the other two using a data collection form master key, teleconference calls occurred, with ongoing queries addressed throughout. Data entry errors were limited by using a web-based data application tool, REDCap, with applicable fields having restrictions and requirements for data entry programmed. Quality assurance reports were performed for each site. Third, our data, which were collected in close proximity to the Canadian licensing of the DOACs (table 1) and subsequent addition to formulary reflect the early uptake of DOACs. Given that our audit year encompassed April 2014 through March 2015, we acknowledge that current day practice patterns may have changed. We aggregated data from different institutions in two cities to compare, overall, the differences in those discharged from the ED versus those hospitalised. The data herein from Edmonton and Regina for the ED cohort ( $n=304$ and $n=83$ patients, respectively) and hospitalised cohort ( $\mathrm{n}=545$ and $\mathrm{n}=120$, respectively) reflect the proportionate populations/catchment areas of these cities. Notably, separate analysis assessing differences among those in the ED and hospitalised cohorts have been performed and presented separately.

\section{CONCLUSION}

In summary, we report distinct differences among patients with acute VTE who are discharged directly from the ED relative to those being hospitalised. Patients hospitalised were older with more concomitant disease, more likely to have PE than DVT, and the PE was more likely to be of higher risk. While DOAC use was similar in both cohorts at just over $25 \%$, variation in a parenteral anticoagulant \pm warfarin was evident between the ED and hospitalised cohorts, and follow-up in the community was influenced based on this regimen. These data support the early uptake of recent advances in VTE management; namely risk stratification of $\mathrm{PE}$ to inform institutional-based use of DOAC therapy. Data, herein, are reflective of practices from two urban cities in Canada with large catchment areas, and therefore offer a broad view of the management of VTE.

Contributors TJB drafted the initial protocol, participated in data acquisition, analysis and interpretation. She also drafted the initial manuscript and approved the final version to be published. BR critically reviewed the research protocol, contributed to the data interpretation, critically revised the manuscript and approved the final version to be published. JB participated in data acquisition, date analysis and interpretation, critically reviewed the manuscript and approved the final version. WS critically reviewed the research protocol, participated in data analysis and interpretation, critically reviewed the manuscript and approved the final version to be published. All authors are accountable for all aspects of the work.

Funding Funding was received from Pfizer Canada (via TJB) in the form of an unrestricted grant.

Disclaimer The sponsor had no role in the protocol design, study conduct, analysis/interpretation of the findings or decision to publish.

Competing interests TJB has received honoraria for an advisory board for Boehringer Ingelheim, as well as honoraria for speaking from Bayer. TJB has received unrestricted grants from Pfizer and Leo Pharma Canada. BR has served on advisory boards, given sponsored lectures using his own slides and received travel expense remuneration for Bayer, Baxter, Beohringer-Ingelheim, CSL-Behring, Pfizer, Sanofi, Servier and Shire. In lieu of honoraria for these activities, the companies have given financial contributions to the University of Alberta. BR reports grants from Novo Nordisk, CSL-Behring and Baxter, all outside of this submitted work. $\mathrm{JB}$ has received speaker honoraria from Boehringer Ingleheim in the past 2 years. WS has received honoraria from Bayer, Pfizer, Bristol Myers Squibb and Boehringer Ingleheim. He has served as a consultant or on an advisory board for Bayer and Pfizer/Bristol Myers Squibb and he has received an unrestricted research grant from Pfizer.

Patient consent Not required.

Ethics approval Health Research Ethics Board approval was received through the University of Alberta (Pro00056384) for Edmonton sites, and the Regina Qu'Appelle Health Region (REB 15-65) for Regina sites.

Provenance and peer review Not commissioned; externally peer reviewed.

Data sharing statement All data within REDCap are at the University of Alberta, and are available to the principal investigator (TJB). No data sharing agreement is in place, hence data sharing is not applicable.

Open access This is an open access article distributed in accordance with the Creative Commons Attribution Non Commercial (CC BY-NC 4.0) license, which permits others to distribute, remix, adapt, build upon this work non-commercially, and license their derivative works on different terms, provided the original work is properly cited, appropriate credit is given, any changes made indicated, and the use is non-commercial. See: http://creativecommons.org/licenses/by-nc/4.0/.

\section{REFERENCES}

1. Wells PS, Forgie MA, Rodger MA. Treatment of venous thromboembolism. JAMA 2014;311:717-28.

2. Kearon C, Akl EA, Comerota AJ, et al. Antithrombotic therapy for VTE disease: antithrombotic therapy and prevention of thrombosis, 9th ed: American College of Chest Physicians Evidence-Based Clinical Practice Guidelines. Chest 2012;141:e419S-94.

3. Kearon C, Akl EA, Ornelas J, et al. Antithrombotic therapy for VTE disease: CHEST guideline and expert panel report. Chest 2016;149:315-52. 
4. Bayer Inc. Xarelto ${ }^{\mathrm{TM}}$ product mongraph. Toronto, Ontario. http://omr. bayer.ca/omr/online/xarelto-pm-en.pdf (accessed 9 Feb 2017).

5. Boehringer Ingelheim Canada Ltd. Pradaxa ${ }^{\mathrm{TM}}$ product monograph, Burlington, Ontario. https://www.boehringer-ingelheim.ca/sites/ca/ files/documents/pradaxapmen.pdf (accessed 9 Feb 2017).

6. Daiichi Sankyo, Inc, Parsippany, NJ, USA (Manufacturer). Progress Therapeutics, Newmarket, Ontario (Importer and Distributer). Lixiana ${ }^{\text {TM }}$ Product Monograph. https://pdf.hres.ca/dpd_pm/ 00037831.PDF (accessed 9 Feb 2017).

7. Pfizer Canada Inc, Kirkland, Quebec and Bristol-Myers Squibb Canada, Montreal, Quebec. Eliquis ${ }^{\mathrm{TM}}$ product monograph. Montreal, Quebec. http://www.pfizer.ca/sites/g/files/g10028126/f/201607/ ELIQUIS_PM_184464_16June2016_E_marketed.pdf (accessed February 9, 2017).

8. Agnelli G, Buller HR, Cohen A, et al. Oral apixaban for the treatment of acute venous thromboembolism. N Engl J Med 2013;369:799-808.

9. Schulman S, Kearon C, Kakkar AK, et al. Dabigatran versus warfarin in the treatment of acute venous thromboembolism. N Engl J Med 2009;361:2342-52

10. Schulman S, Kakkar AK, Goldhaber SZ, et al. Treatment of acute venous thromboembolism with dabigatran or warfarin and pooled analysis. Circulation 2014;129:764-72.

11. Bauersachs R, Berkowitz SD, Brenner B, et al. Oral rivaroxaban for symptomatic venous thromboembolism. N Engl J Med 2010;363:2499-510.

12. Büller HR, Prins $M H$, Lensin AW, et al. Oral rivaroxaban for the treatment of symptomatic pulmonary embolism. $N$ Engl J Med 2012;366:1287-97.

13. Büller HR, Décousus H, Grosso MA, et al. Edoxaban versus warfarin for the treatment of symptomatic venous thromboembolism. $N$ Engl $J$ Med 2013;369:1406-15.

14. Konstantinides SV, Torbicki A, Agnelli G, et al. 2014 ESC guidelines on the diagnosis and management of acute pulmonary embolism. Eur Heart J 2014;35:3033-73.

15. Population of Edmonton area. http://globalnews.ca/news/1824910/ edmonton-region-has-canadas-2nd-highest-population-growthstats-canada/ (accessed 31 Oct 2017).
16. Population of Regina. http://www.leaderpost.com/life/Regina+ population+cracks/9553267/story.html (accessed 31 Oct 2017).

17. Chan CM, Woods C, Shorr AF. The validation and reproducibility of the pulmonary embolism severity index. $J$ Thromb Haemost 2010;8:1509-14.

18. Righini M, Roy PM, Meyer G, et al. The Simplified Pulmonary Embolism Severity Index (PESI): validation of a clinical prognostic model for pulmonary embolism. J Thromb Haemost 2011;9:2115-7.

19. Bungard TJ, Ritchie B, Bolt J, et al. Management of acute venous thromboembolism among a cohort of patients discharged directly from the emergency department. BMJ Open 2018;0:e022064.

20. Bungard TJ, Ritchie B, Bolt J, et al. Anticoagulant therapies for acute venous thromboembolism among a cohort of patients discharged from Canadian urban and rural hospitals. BMJ Open 2018;0:e022065.

21. Piran S, Le Gal G, Wells PS, et al. Outpatient treatment of symptomatic pulmonary embolism: a systematic review and metaanalysis. Thromb Res 2013;132:515-9.

22. Vinson DR, Zehtabchi S, Yealy DM. Can selected patients with newly diagnosed pulmonary embolism be safely treated without hospitalization? A systematic review. Ann Emerg Med 2012:60:651-62.

23. Zondag W, Kooiman J, Klok FA, et al. Outpatient versus inpatient treatment in patients with pulmonary embolism: a meta-analysis. Eur Respir J 2013;42:134-44.

24. Singer AJ, Thode HC, Peacock WF. Admission rates for emergency department patients with venous thromboembolism and estimation of the proportion of low risk pulmonary embolism patients: a US perspective. Clin Exp Emerg Med 2016;3:126-31.

25. Vinson DR, Ballard DW, Huang J, et al. Timing of discharge follow-up for acute pulmonary embolism: retrospective cohort study. West $J$ Emerg Med 2015;16:55-61.

26. Dault R, Vanasse A, Blais L, et al. Patterns and predictors of use of anticoagulants for the treatment of venous thromboembolism following approval of rivaroxaban. Clin Appl Thromb Hemost 2016;22:765-71. 